\title{
Breast involvement in immunolymphoproliferative disorders: Report of a case of multiple myeloma of the breast.
}

\author{
Arpit Sharma, B K Sharma, Ashish Bakliwal
}

\begin{abstract}
Summary: Breast involvement by immunolymphoproliferative disordersis rare. Primary and secondary malignant lymphomas of the breast are much more common than multiple myeloma, of which only a few cases have been described. We report a case of a patient with an established diagnosis of multiple myeloma in whom breast involvement appeared after the completion of the treatment. We underline diagnostic difficulties caused by the lack of clinical and radiological features which allow us to differentiate between breast carcinoma and breast involvement by lymphoproliferative disease. Only fine needle aspiration and/or excisional biopsy can differentiate between immunolymphoproliferative

disorders and epithelial or mesenchymal tumors of the breast.
\end{abstract}

\section{Introduction}

Multiple myeloma(MM) is the most frequent lympho-immunoproliferative disorders, but their localization in the breast is quite rare.

MM is a disseminated malignant B-cell lineage neoplasm, characterized by clonal proliferation of plasma cells in the bone marrow, associated with the overproduction of structurally homogeneous immunoglobulins (M-component). They represent the most frequent form of plasma cell dyscrasias and about $1 \%$ of all types of tumors [1]. The incidence increases progressively with

age and the mean age at diagnosis is about 65 years, while the onset in children [2] and young adults before 40 years is very rare [3]. Impaired hemopoiesis, renal failure, osteolytic bone lesions, recurrent infections, hypercalcemia are the most common features of the disease. Clinically apparent extraosseous manifestation are present in less than $5 \%$ of patients with multiple myeloma

and are usually associated with more aggressive behaviour, resistance to treatment and shorter survival. Clinicopathologic studies, however, show extraosseous involvement in about two third of the patients, liver, spleen and lymph nodes being the most frequent sites $[4,5]$. Central nervous system, kidney, skin, pleura, lung, testes, pancreas, thyroid, adrenal glands and omental

involvement have also been described. Few cases of breast multiple myeloma are reported in

the literature [2, 6-14]. Ross et al. [15] described one case and reviewed 10

cases of multiple myeloma of the breast reported up to 1987. Furthermore, one case by Collins et al. [16] and two cases by Moulopoulos et al. [17] have been reported, the latter only as imaging features without any further information. More recently two cases have been described by Kim

[18] and Ariad [19].

In this report we describe the case of a patient with established diagnosis of multiple myeloma treated with cytotoxic combination chemotherapy who developed breast involvement after completing the course of treatment of the disease. We discuss their initial management.

Case report

A 47-year-old man was diagnosed to have IgG $(k)$ MM stage IIIA in 2010, and was treated with alternating

cycles of VAD/MP (vincristine, adriamycin, dexamethazone-melphalan, prednisone) for a total of eight courses.After which he stopped treatment on the advice of the treating doctor and a normal electrophoresis

.In 2013the patient felt a mass in the right breast. At clinical examination there was a single palpable mass in the right $(4.4 \times 2.8 \mathrm{~cm})$ Mammography showed single nodular mass. Ultrasound examination of the breast showed solid echo-poor mass with regular margins. Fine needle aspiration cytology showed immature nucleolated plasma cells. Excisional biopsy was performed, histological examination confirming the myelomatous nature of the mass. Microscopically the nodules were composed of tightly packed immature plasma cells infiltrating the stroma. 

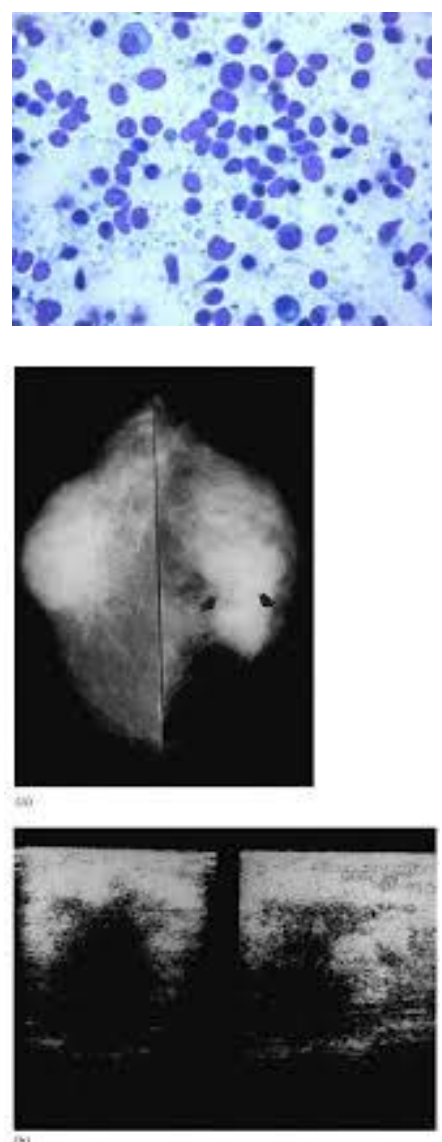

Some res idual lobular glands were evident focally throughout the neoplastic infiltration.

Biopsy revealed plasma cell tumor with eccentric atypical nuclei and abundant cytoplasm showing peri-nuclear halo,suggestive of metastatic deposits from multiple myeloma.

Immunohistochemistry revealed positivity for KAPPA LIGHT CHAIN IHC,CD138 and KI67 confirming the diagnosis of Plasmacytoma. 


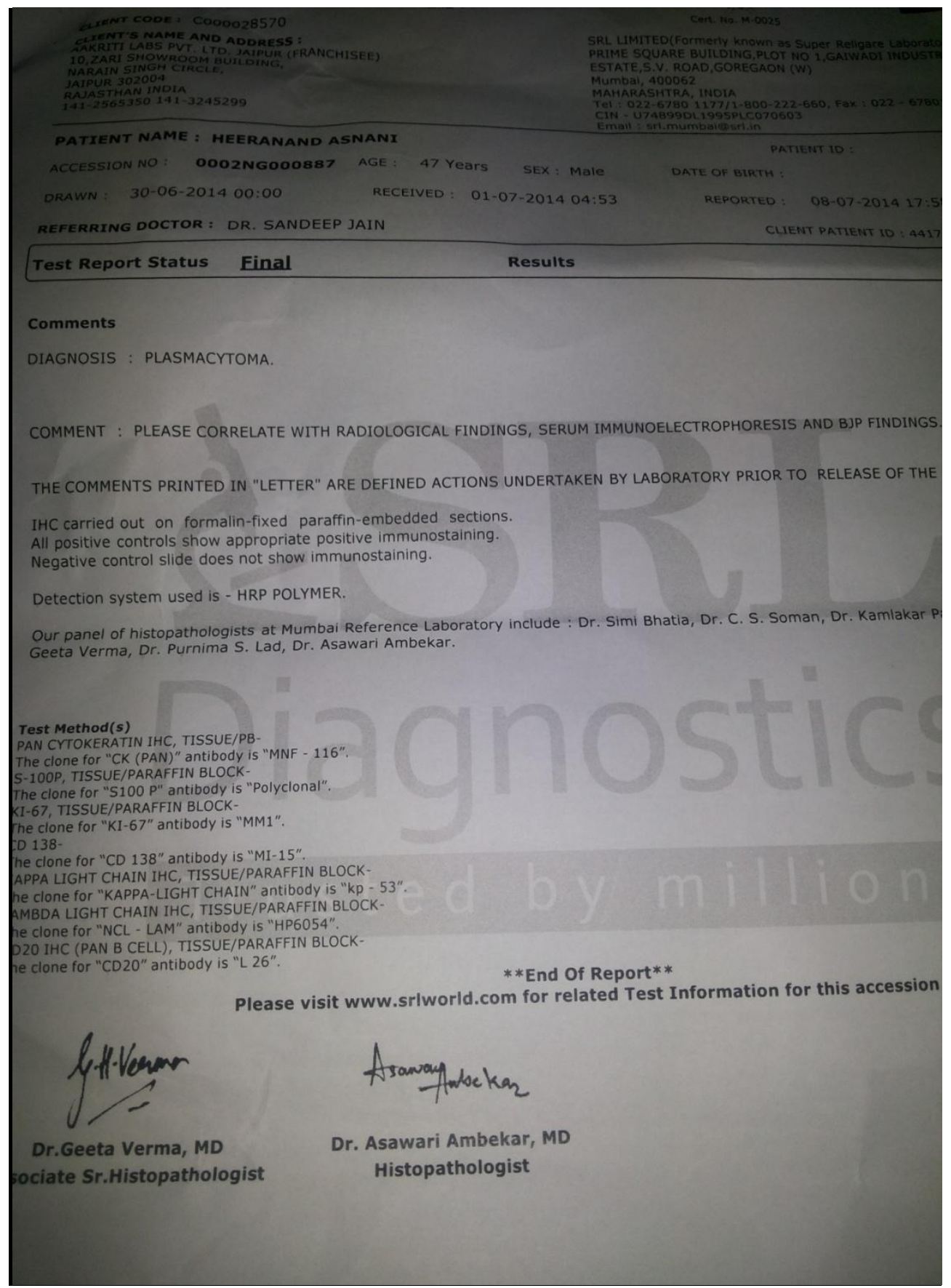




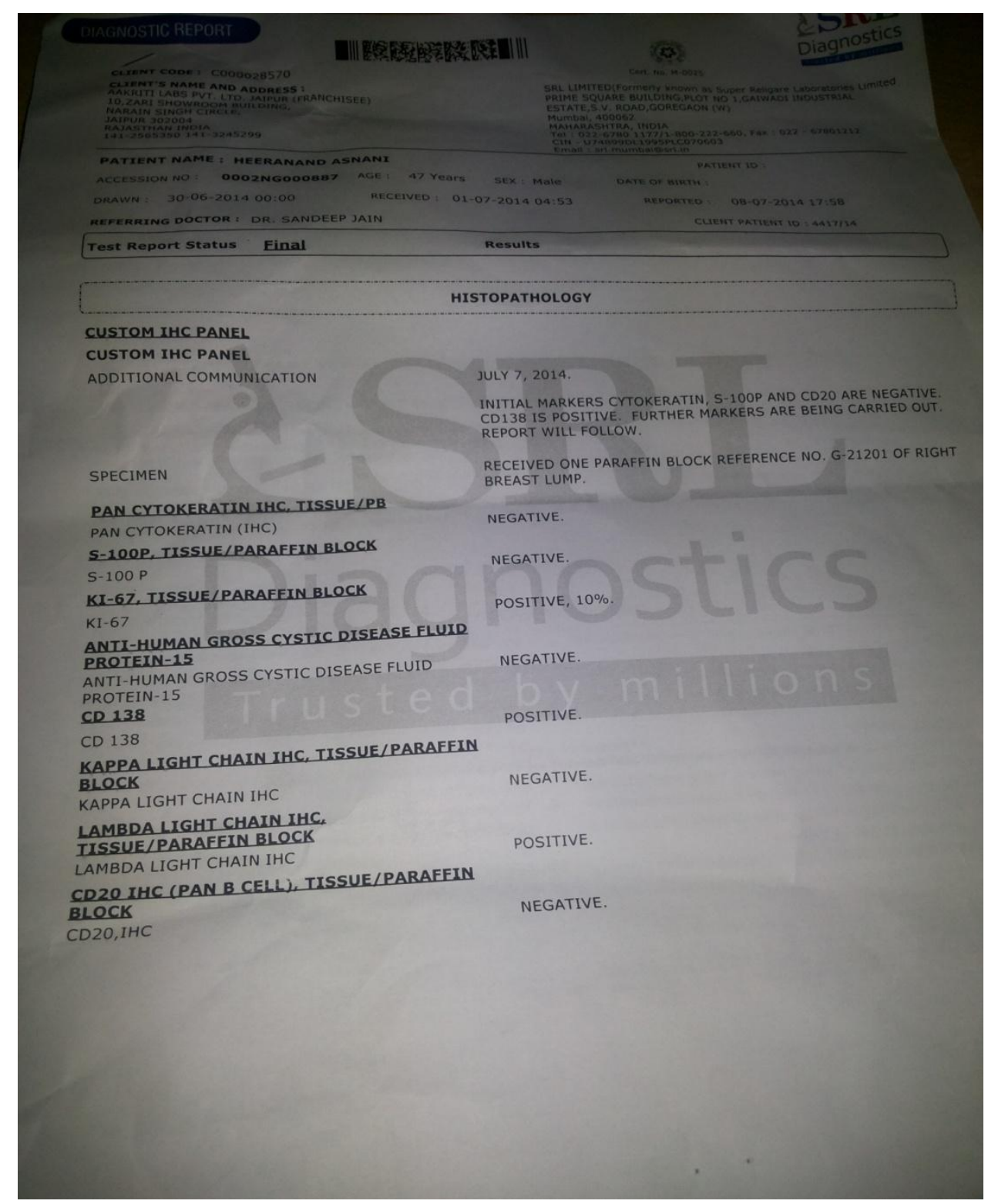

\section{Discussion;}

Ductal and lobular carcinoma are the most frequent Imalignant disease of the breast, while lymphoproliferative

disorders and mesenchymal tumors are exceedingly rare. Patients affected by immunolymphoproliferative disorders, particularly when treated with chemotherapy, may develop secondary tumor in the breast. The appearance of breast nodules in patients with an immunoproliferative disorders make it mandatory to differentiate between primary breast cancer and a haematological malignancy. Multiple myeloma is a systemic disease which may involve extraosseous sites, but only in rare occasions has it been found in the breast. To date, we have only found very few case reports describing multiple myeloma involving the breast. Analysis of data reported in the literature are consistent with a lack of clinical or instrumental features that allow us to differentiate between multiple myeloma involving the breast and breast carcinoma. In particular, mammography and ultrasound examination does not help the differential diagnosis. The mammographic pattern of multiple myeloma is generally characterized by round or lobulated well denned nodules mimicking a benign process without microcalcification. Recently Collins et al. [16] described one case of breast multiple myeloma using ultrasound and color Doppler examination. The presence of an echo-poor mass with posterior acoustic shadowing and the presence of neovascularization was similar to those found in presence of carcinoma.

In our case the ultrasound images showed relatively echo-poor mass with rather distinct margins and posterior reinforcement. The mammographic pattern was that of nodules with well-defined margins without parenchymal distorsion .Plasma cells tumors may appear either in a solitary form or as disseminated multiple myeloma. Extramedullary plasmocytomas are rare and can represent either the initial manifestation of systemic MM 
or remain solitary for long time. Among the five patients with solitary breast plasmocytoma in two cases the localization was bilateral [7, 12], whereas in the other three it appeared to be a 'true' solitary plasmocytoma $[10,13,14]$. Only in one case was the breast involvement associated with a serum monoclonal gammopathy which disappeared after local treatment [14]. In all the three cases no recurrence has been shown after 40 months [14], 46 months [10] and 9 years [13] at the time of pubblication. The usual treatment of extramedullary plasmocytoma is radiotherapy with excellent long time results. The treatment of solitary plasmocytoma of the breast should consist of local excision followed by radiation therapy, whereas when breast involvement is secondary to disseminated MM the treatment should be that of the basic disease employing the most widely used poly chemotherapy schedules like VAD (vincristine, adriamycine, dexamethazone) or MP (melphalan, 6-methilprednisolone).

\section{References}

[1]. Kyle RA. Multiple myeloma. An update on diagnosis and management. Acta Oncol 1990; 29: 1-8.

[2]. Maeda K, Abesamis CM, Kuhn LM, Hyun BH. Multiple myeloma in childhood: Report of a case with breast tumors as a presenting manifestation. Am J Clin Pathol 1973; 60: 552-8.

[3]. Hewell GM, Alexanian R. Myeloma in young persons. Ann Intern Med 1976; 84: 441-8.

[4]. Kapadia SB. Multiple myeloma: A clinicopathologic study of 62 consecutively autopsied cases. Medicine 1980; 59: 380-92.

[5]. Pasmantier MW, Azar HA. Extraskeletal spread in multiple plasma cell myeloma. Cancer 1969; 23: 167-74.

[6]. Cutler CW. Plasma cell tumor of the breast with metastases. Ann Surg 1934; 100: 392-5.

[7]. Innes J, Newall J. Myelomatosis. Lancet 1961; 1: 239-45.

[8]. Rosenberg B, Attie JN, Mandelbaum HL. Breast tumor as the presenting sign of multiple myeloma. N Engl J Med 1963; 269: 35961 .

[9]. Craft IL. The late appearance of extramedullary lesion in myelomatosis. Br J Cancer 1967; 21: 501-4.

[10]. Proctor NSF, Rippey JJ, Shulman G, Cohen C. Extramedullary plasmocytoma of the breast. J Pathol 1975; 116: 97-100.

[11]. Bassett WB, Weiss RB. Plasmocytoma of the breast: An unusual manifestation of multiple myeloma. South Med J 1979; 72: 14924.

[12]. Bloomberg TJ, Glees JP, Williams JE. Bilateral breast lumps: An unusual feature of extramedullary plasmocytoma. Br J Radiol1980; 53: 498-501.13. Merino MJ. Plasmocytoma of the breast. Arch Pathol Lab Med1984; 108: 676-8.

[13]. Kirshenbaum G, Rhone DP. Solitary extramedullary plasmocytoma of the breast with serum monoclonal protein: A case report and review of the literature. Am J Clin Pathol 1985; 83: 230-2.

[14]. Ross JS, King TM, Spector JI et al. Plasmocytoma of the breast. An unusual case of recurrent myeloma. Arch Intern Med 1987; 147:1838-40.

[15]. Collins CD, Kedar RP, Cosgrove DO. Case report: Myeloma of the breast - appearances on ultrasound and color Doppler. Br J Radiol 1994; 67: 399-400.

[16]. Moulopoulos LA, Grandfield CA, Dimopoulos MA et al. Extraosseous multiple myeloma. Imaging features. Am J Roentgenol 1993; 161: 1083-7.

[17]. Kim EE, Sawwaf ZU, Sneigej N. Multiple myeloma of the breast: Magnetic resonance and ultrasound imaging findings. Breast Dis 1996; 9: 229-33.

[18]. Ariad S, Lewis D, Cohen R, Bezwoda WR. Breast Lymphoma. A clinical and pathological review and 10-year treatment results. South Afr Med J 1995; 85: 85-9.

[19]. Mambo NC, Burke JS, Butler JJ. Primary malignant lymphomas of the breast. Cancer 1977; 39: 2033-40.

[20]. Wiseman C, Liao KT. Primary lymphoma of the breast. Cancer 1972; 29: 1705-12.

[21]. Freeman C, Berg JW, Cutler SJ. Occurrence and prognosis of extranodal lymphomas. Cancer 1972; 29: 252-60.

[22]. Navas JJ, Battifora H. Primary lymphoma of the breast: Ultrastructural study of two cases. Cancer 1977; 39: 2025-32

[23]. Cohen PL, Brooks JJ. Lymphomas of the breast. A clinicopathologic and immunohistochemical study of primary and secondary cases. Cancer 1991; 67: 1359-69.

[24]. Jeon HJ, Akagi T, Hoshida Yet al. Primary malignant lymphoma of the breast. An immunohistochemical study of seven patients and literature review of 152 patients with breast lymphoma in Japan. Cancer 1992; 70: 2451-9.

[25]. Hugh JC, Jackson FI, Hanson J, Poppema S. Primary breast lymphoma. An immunohistologic study of 20 new cases. Cancer 1990; 66: 2602-11.

[26]. Brustein S, Kimmel M, Lieberman PH et al. Malignant lymphoma of the breast. A study of 53 patients. Ann Surg 1987; 205: 14450 .

[27]. 29. Meyer JE, Kopans DB, Long JC. Mammographic appearance of malignant lymphoma of the breast. Radiology 1980; 135: 6236.

[28]. 30. Carrie C, Noel E, Billy C et al. Primary non-Hodgkin lymphomaof the breast. A follow-up of 14 cases from 1971 to 1985. Breast DisSenol 1985; 1: 165-71.

[29]. 31. Dixon JM, Lumsden AB, Elton RA, Anderson TJ. Primary lymphoma of the breast. Br J Surg 1987; 74: $214-7$.

[30]. 32. Panzarola P, Bosso P, Stocchi A. II linfoma mammario. Radiat Med 1994; 87: 250-3. 